05

\title{
Управление спектром излучения диодного лазера при ОВЧ-модуляции тока инжекции
}

\author{
(C) К.Н. Савинов ${ }^{1}$, А.К. Дмитриев ${ }^{1,2}$, А.А. Рундау ${ }^{3}$ \\ ${ }^{1}$ Новосибирский государственный технический университет, \\ 630073 Новосибирск, Россия \\ ${ }^{2}$ Институт лазерной физики Сибирского отделения РАН, \\ 630090 Новосибирск, Россия \\ ${ }^{3}$ Сибирский государственный университет геосистем и технологий, \\ 630108 Новосибирск, Россия \\ e-mail: K.N.Savinov@yandex.ru
}

Поступила в редакцию 14.09.2020 г.

В окончательной редакции 16.02.2021 г.

Принята к публикации 26.02.2021 г.

Представлены результаты исследований механизмов управления интервалами между парами ОВЧ-компонент на боковых полосах спектра вблизи частоты часового перехода рубидия.

Ключевые слова: модуляция в диапазоне очень высоких частот (ОВЧ), спектр диодного лазера, резонанс когерентного пленения населенностей (КПН), световой сдвиг.

DOI: $10.21883 /$ OS.2021.06.50988.222-20

\section{Введение}

Работы по повышению стабильности квантовых стандартов частоты проводятся непрерывно с момента создания мазеров и лазеров [1]. В качестве реперов для квантовых стандартов частоты служат узкие спектральные резонансы. Так, использование запрещенного перехода в атоме ${ }^{133} \mathrm{Cs}$ на частоте $9.192 \mathrm{GHz}$ позволило достичь погрешности воспроизведения частоты первичного стандарта порядка $10^{-16}$ [2], а переход к оптическому и более коротковолновому диапазонам открывает перспективы для повышения стабильности стандартов [3].

Вместе с тем для широкого круга задач достаточно точности измерений на уровне $10^{-12}$, что обеспечивается доступными рубидиевыми часами с использованием в качестве частотного репера резонанса когерентного пленения населенностей (КПН), который сначала был предсказан [4], а затем практически сразу же был зарегистрирован в ячейке с атомами натрия [5]. Что касается рубидиевых стандартов частоты, минимальными сдвигами обладает сверхтонкий переход 5S1/2F2-5S1/2F1 основного состояния D1 линии изотопа рубидия-87 на длине волны $795 \mathrm{~nm}$ [6].

В КПН-резонансе используются два оптических поля, которые взаимодействуют с электронными переходами в щелочных атомах, таких как Rb. Разность частот между этими полями равна сверхтонкому расщеплению основного электронного состояния атомов. Эти два поля оптически перекачивают атомы в невзаимодействующую когерентную суперпозицию двух сверхтонких состояний („темное состояние “).

В настоящее время для накачки рубидиевых часов широко используются лазеры с вертикальным резонатором, преимущество которых заключается в низком уровне потребления энергии и малых габаритах [7,8]. Вместе с тем малые габариты позволяют эффективно модулировать спектр излучения этого лазера в широком интервале сверхвысоких частот (СВЧ), в том числе и на частоте часового перехода. Однако ширина спектра подобных лазеров составляет величину порядка $50 \mathrm{MHz}$ [9], что является препятствием для их применения в оптических стандартах частоты. В то же время полупроводниковые лазеры с внешним резонатором обладают существенно меньшей шириной линии (порядка $60 \mathrm{kHz}$ ) [10]. Это открывает возможность создания комбинированного рубидиевого стандарта частоты с накачкой диодным лазером при одновременной стабилизации его частоты по оптическому резонансу, а частоты модуляции тока инжекции лазера - по КПН-резонансу.

Оптическая накачка КПН-резонансов в атомах рубидия с использованием одного лазера была осуществлена при синусоидальной модуляции тока инжекции $\mathrm{AlGaAs}$ лазерного диода на длине волны излучения $780 \mathrm{~nm}$ [11]. При субгармонической частотной модуляции на $1.139 \mathrm{GHz}$ с индексом модуляции 4.2 на частоте часового перехода $F_{0}$, равном $6.834682613 \mathrm{GHz}$, был зарегистрирован резонанс шириной $3 \mathrm{kHz}$.

Вместе с тем была продемонстрирована эффективная СВЧ-накачка в диодном лазере на более высоких частотах (порядка $3.4 \mathrm{GHz}$ ), когда разность между боковыми составляющими спектра совпадает с частотой часового перехода $F_{0}[12]$. Однако наблюдавшееся различие амплитуд боковых составляющих будет приводить к световым сдвигам.

Для уменьшения этого сдвига было предложено использовать широкий спектр частот фемтосекундного ла- 
зера, частота повторений импульсов которого привязана к частоте $F_{0}[13]$. Однако, поскольку вклад в образование КПН-резонанса вносят только те частоты, которые попадают в спектр оптических переходов, отношение сигнал/шум при наблюдении резонанса с накачкой фемтосекундным лазером будет чрезвычайно низким, что, повидимому, и объясняет отсутствие экспериментальных работ в этом направлении.

В диодном лазере с межмодовым интервалом резонатора порядка $3.4 \mathrm{GHz}$ модуляция тока инжекции в диапазоне очень высоких частот (ОВЧ) позволила получить многочастотный спектр на обеих боковых полосах при одновременном достижении равенства их амплитуд [14].

Однако вопрос об управлении частотными интервалами между соответствующими компонентами ОВЧ-структур различных боковых полос спектра (вблизи $F_{0}$ ) остался открытым.

В настоящей работе приведены результаты исследований механизмов управления интервалами между парами ОВЧ-компонент на боковых полосах спектра вблизи частоты часового перехода рубидия.

\section{Экспериментальная установка}

В работе использовался полупроводниковый лазер с внешним резонатором, пороговый ток которого составил $49 \mathrm{~mA}$. Оптическая длина резонатора $44 \mathrm{~mm}$, что соответствует межмодовому интервалу $3.4 \mathrm{GHz}$. Основными элементами лазера являются лазерный диод, микролинза и дифракционная решетка, закрепленная на пьезокерамике (подробное описание содержится в работе [12]). В качестве гетеродинного использовался аналогичный лазер с пороговым током $55 \mathrm{~mA}$.

Блок-схема экспериментальной установки представлена на рис. 1.

Сигналы от источника постоянного тока (1) и высокочастотного (ВЧ) генератора (3) через смеситель (2) поступали на исследуемый лазер (5). Для регистрации спектра в широком интервале частот излучение через оптический изолятор (8) направлялось на сканируемый интерферометр Фабри-Перо (14) с областью свободной дисперсии порядка $20 \mathrm{GHz}$. Пропускания входного и выходного зеркал составили 5\% и 40\% соответственно. Выходное зеркало интерферометра было прикреплено к пьезокерамическому преобразователю (13), который использовался для сканирования длины интерферометpa c помощью цифрового генератора пилообразного напряжения (16). Для удобства записи спектров входное зеркало крепилось на другом пьезокерамическом преобразователе (13), длина которого управлялась при помощи источника постоянного напряжения (12), что позволяло располагать исследуемые спектры в центральной части экрана осциллографа. После интерферометра излучение поступало на фотодетектор (17), с выхода которого сигнал подавался на цифровой осциллограф (18). На другой вход осциллографа поступал

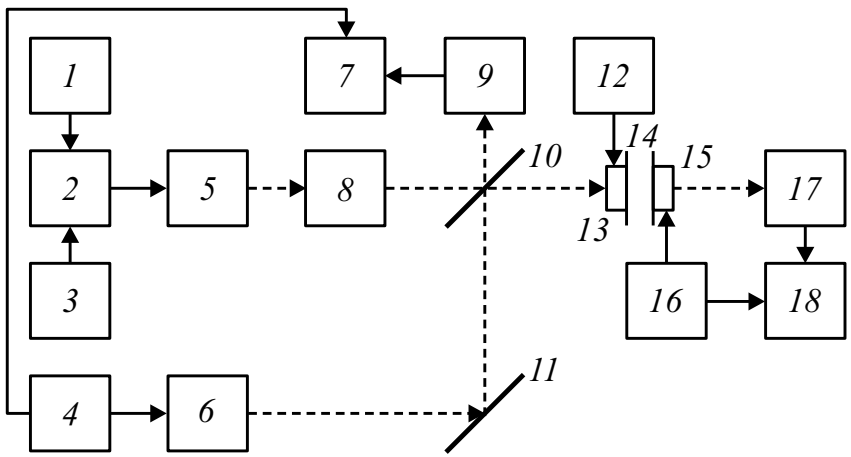

Рис. 1. Схема измерений спектра излучения лазера: 1 - источник постоянного тока; 2 - смеситель; 3 - ВЧ-генератор; 4,16 - генераторы пилообразного напряжения; 4 - смеситель; 5 - исследуемый лазер; 6 - гетеродинный лазер; 7,18 - цифровые осциллографы; 8 - оптический изолятор; 9,17 - фотодетекторы; 10 - полупрозрачное зеркало, 11 поворотное зеркало; 12 - источник постоянного напряжения; 13,15 - пьезокерамические преобразователи; 14 - сканируемый интерферометр Фабри-Перо. Штриховые линии световые пучки, сплошные линии - электрические связи.

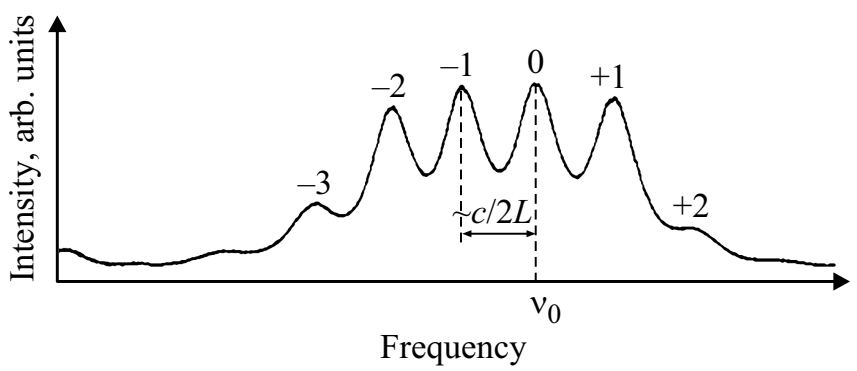

Рис. 2. Спектры излучения лазера при ВЧ-модуляции частотой $85 \mathrm{MHz}$ с амплитудой тока $5.5 \mathrm{dBm}$. $v_{0}$ - частота лазера в отсутствие модуляции.

также сигнал от генератора пилообразного напряжения (16).

Для изучения тонкой структуры спектра использовался гетеродинный полупроводниковый лазер (6), излучение которого через систему зеркал $(10,11)$ смешивалось со световым пучком исследуемого лазера и поступало на фотоприемник (9) и сканируемый интерферометр Фабри-Перо (14). Частота гетеродинного лазера сканировалась подачей сигнала от генератора пилообразного напряжения (4) с периодом $40 \mathrm{~ms}$ на пьезокерамику резонатора. Напряжение с фотоприемника (9) подавалось на цифровой осциллограф (7), на другой вход которого поступал сигнал от генератора пилообразного напряжения (4). Для регистрации биений на различных полосах спектра частота излучения гетеродинного лазера перестраивалась с помощью изменения тока инжекции. 
$a$

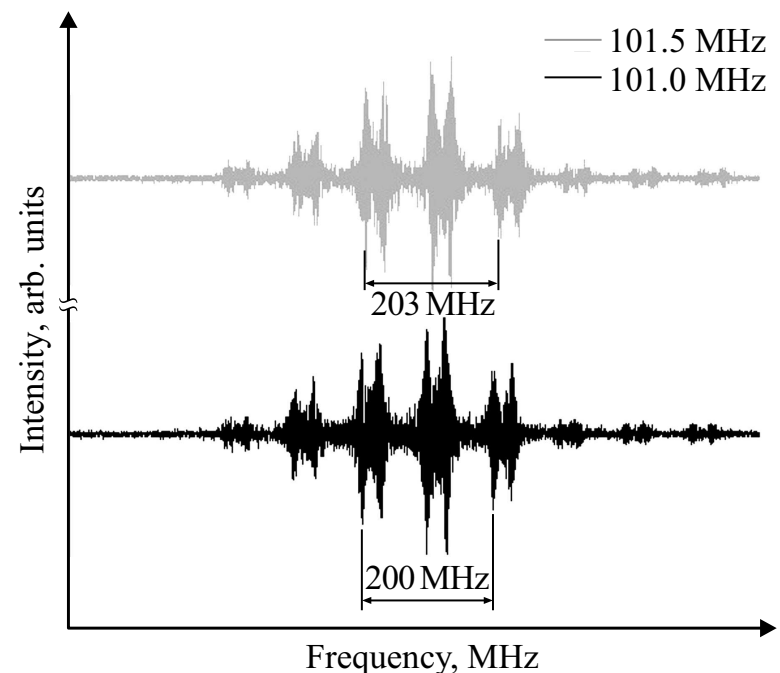

$b$

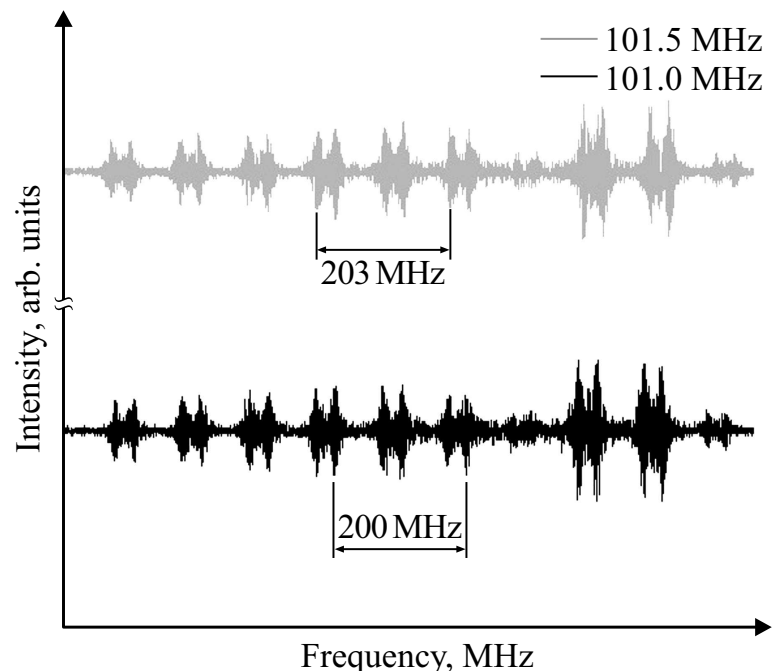

Рис. 3. Биения на НЧ $(a)$ и ВЧ $(b)$ боковых полосах спектра при ОВЧ-модуляции с частотами 100 и $101.5 \mathrm{MHz}$.

\section{Экспериментальные результаты и их обсуждение}

Измерения проводились при токе инжекции $52 \mathrm{~mA}$. Спектр излучения лазера при ОВЧ-модуляции частотой $85 \mathrm{MHz}$ представлен на рис. 2. Для удобства все полосы пронумерованы: нулевая соответствует несущей в отсутствие ОВЧ-сигнала, высокочастотные боковые полосы спектра обозначены знаком „++“, низкочастотные - знаком „-““. Частотный интервал между соседними полосами спектра составляет величину, близкую к межмодовому интервалу.

Более детально спектр при различных частотах модуляции и токах инжекции был исследован в работе [14]. Также в этой работе было предположено, что ОВЧ-структура спектра во всем диапазоне излучения лазера определяется формулой

$$
v_{m}=v_{0}+m v_{\text {mod }}
$$

где $v_{m}$ - частота $m$-й ОВЧ-компоненты, $v_{0}$ - частота лазера в отсутствие модуляции, $v_{\bmod }$ - частота модуляции и $m-$ порядок модуляции.

Предполагалось, что интервал между выбранными парами компонент, величина которого близка к частоте часового перехода, может регулироваться частотой модуляции.

При подаче ОВЧ-модуляции сначала возникает ОВЧ-структура на несущей полосе спектра, при этом излучение присутствует только вокруг одной моды. Начиная с мощности модуляции $-8 \mathrm{dBm}$ возникает генерация на соседних модах и, причем сразу, наблюдается ОВЧ-структура. При увеличении тока накачки до $54 \mathrm{~mA}$ порог возникновения боковых полос возрастает до $-4 \mathrm{dBm}$.

Для того чтобы проверить связь между компонентами спектра во всем диапазоне генерации, были использова- ны две частоты модуляции: 100 и $101.5 \mathrm{MHz}$. Согласно формуле (1), для $m=34$ (центр боковых полос) смещение ОВЧ-структур спектра на боковых полосах составит $51 \mathrm{MHz}$, при этом структуры на исследуемых частотах будут максимально смещены относительно друг друга.

На рис. 3 показан сравнительный спектр излучения лазера при частотах модуляции 100 и $101.5 \mathrm{MHz}$. На ВЧполосе наблюдается существенно большее количество ОВЧ-компонент, что может быть связано с нелинейными процессами.

Экспериментально измеренное отношение интервалов между компонентами спектра как для низкочастотных (НЧ), так и для ВЧ-полос составило $1.018 \pm 0.005$, что, как и следовало ожидать, согласуется с отношением частот модуляции (1.015). Интервал между ОВЧ-компонентами на боковых полосах с учетом погрешности изменяется на величину менее $10 \mathrm{MHz}$, что не согласуется с формулой (1). Это означает, что частота ОВЧ-модуляции напрямую не влияет на интервалы между ОВЧ-компонентами на различных полосах спектра.

Для того чтобы выяснить связь между компонентами различных полос спектра излучения лазера при ОВЧ-модуляции, были записаны биения на боковых полосах при различных частотах модуляции (рис. 4).

Видно, что положение одной из ОВЧ-компонент, которую можно считать „центральной“, на каждой из боковых полос остается неизменным (штриховая линия) с погрешностью $\pm 5 \mathrm{MHz}$, в то время как интервалы между ОВЧ-компонентами определяются частотой модуляции. Погрешность измерений связана с флуктуациями и тепловым дрейфом длины резонатора. Такая ситуация возможна, когда ОВЧ-структура формируется вокруг определенных мод резонатора. При этом управление положением ОВЧ-структуры на боковых полосах спектра возможно за счет изменения длины резонатора. 

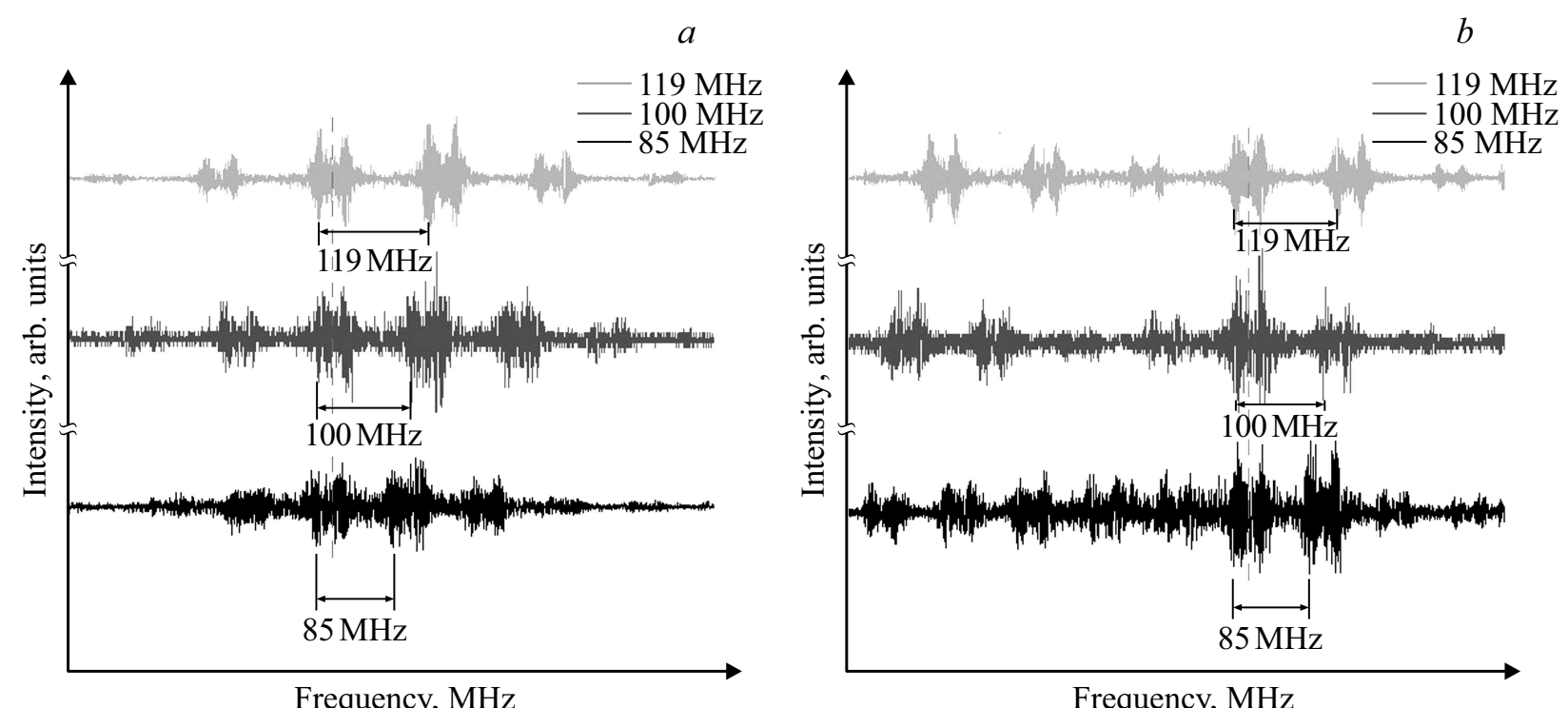

Рис. 4. Биения на боковых компонентах при различных частотах ОВЧ-модуляции.

\section{Заключение}

Обнаружено, что многочастотная структура, задаваемая ОВЧ-модуляцией, формируется вокруг мод внешнего резонатора диодного лазера. Частотный интервал между соответствующими парами ОВЧ-компонент, дающими вклад в КПН-резонанс, может регулироваться длиной резонатора.

\section{Финансирование работы}

Работа выполнена при поддержке Министерства науки и высшего образования РФ, проект № FSUN-2020-0007 и гранта Российского фонда фундаментальных исследований № 18-02-00316.

\section{Конфликт интересов}

Авторы заявляют, что у них нет конфликта интересов.

\section{Список литературы}

[1] Quinn T. // Metrologia. 2005. V. 42. N 3. doi 10.1088/00261394/42/3/E01

[2] Bize S., Laurent P., Abgrall M., Marion H., Maksimovic I., Cacciapuoti L., Grunert J., Vian C., Pereira dos Santos F., Rosenbusch P., Lemonde P., Santarelli G., Wolf P., Clairon A., Luiten A., Tobar M., Salomon C. // J. Phys. B: At. Mol. Opt. Phys. 2005. V. 38. N 9. P. 449. doi 10.1088/09534075/38/9/002

[3] Hollberg L., Oates C.W., Wilpers G., Hoyt C.W., Barber Z.W., Diddams S.A., Oskay W.H., Bergquist J.C. // J. Phys. B: At. Mol. Opt. Phys. 2005. V. 38. N 9. P. 469. doi 10.1088/09534075/38/9/003

[4] Arimondo E., Orriols G. // Lettere Al Nuovo Cimento. 1976. V. 17. P. 333. doi 10.1007/BF02746514
[5] Alzetta G., Gozzini A., Moi M., Orriols G. // Il Nuovo Cimento. 1976. V. 36. P. 5. doi 10.1007/BF02749417

[6] Stähler M., Wynands R., Knappe S., Kitching J., Hollberg L., Taichenachev A., Yudin V. // Optics Lett. 2002. V. 27. N 16. P. 1472. doi 10.1364/OL.27.001472

[7] Knappe S., Schwindt P., Shah V., Hollberg L., Kitching J., Liew L., Moreland J. // Opt. Express. 2005. V. 13. N 4. P. 1249. doi 10.1364/OPEX.13.001249

[8] Хрипунов С.А., Раднатаров Д.А., Кобиев С.М., Юдин В.И., Тайченачев А.В., Басалаев М.Ю., Балабас М.В., Андрюшков В.А., Попков И.Д. // Квант. электрон. 2016. Т. 46. № 7. C. 668; Khripunov S.A., Radnatarov D.A., Kobtsev S.M., Yudin V.I., Taichenachev A.V., Basalaev M.Yu., Balabas M.V., Andryushkov V.A., Popkov I.D. // Quantum Electronics. 2016. V. 46. N 7. P. 668. doi $10.1070 \% 2 F Q E L 15992$

[9] Kitching J., Knappe S., Vukicevic N., Hollberg L., Wynands R., Weidmann W. // IEEE Trans. Instrum. Meas. 2000. V. 49. N 6. P. 1313. doi 10.1109/19.893276

[10] Vassiliev V.V., Zibrov S.A., Velichansky V.L. // Rev. Sci. Instrum. 2006. V. 77. N 1. P. 013102. doi 10.1063/1.2162448

[11] Cyr N., Tětu M., Breton M. // IEEE Trans. Instrum. Meas. 1993. V. 42. N 2. P. 640. doi 10.1109/19.278645

[12] Исакова А.А., Савинов К.Н., Головин Н.Н., Алтынбеков Н.Ж., Вишняков В.И., Дмитриев А.К. // Квант. электрон. 2017. Т. 47. № 7. С. 610; Isakova A.A., Savinov K.N., Golovin N.N., Altynbekov N.Zh., Vishnyakov V.I., Dmitriev A.K. // Quantum Electron. 2017. V. 47. N 7. P. 610. doi $10.1070 \% 2$ FQEL16407

[13] Baklanov E.V., Dmitriev A.K. // Laser Physics. 2010. V. 10. P. 52. doi 10.1134/S1054660X09170010

[14] Исакова А.А., Головин Н.Н., Савинов К.Н., Дмитриев А.К. // Квант. электрон. 2019. Т. 49. № 6. C. 600; Isakova A.A., Golovin N.N., Savinov K.N., Dmitriev A.K. // Quantum Electron. 2019. V. 49. N 6. P. 600. doi $10.1070 \% 2$ FQEL16889 